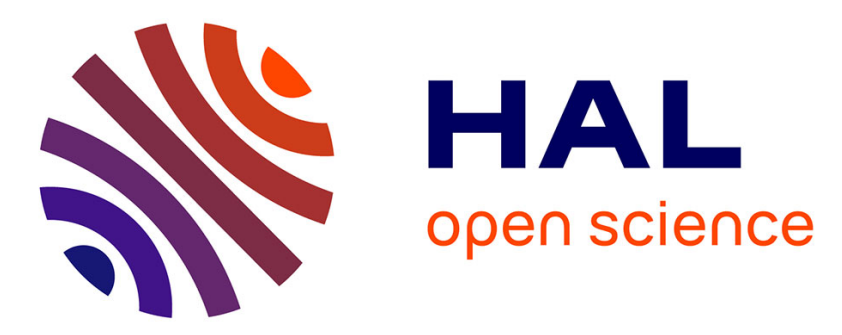

\title{
A solidification model for the columnar to equiaxed transition in welding of a Cr-Mo ferritic stainless steel with Ti as inoculant
}

Vincent Villaret, Frédéric Deschaux-Beaume, Cyril Bordreuil

\section{- To cite this version:}

Vincent Villaret, Frédéric Deschaux-Beaume, Cyril Bordreuil. A solidification model for the columnar to equiaxed transition in welding of a $\mathrm{Cr}$-Mo ferritic stainless steel with $\mathrm{Ti}$ as inoculant. Journal of Materials Processing Technology, 2016, 233, pp.115-124. 10.1016/j.jmatprotec.2016.02.017 . hal01288900

\author{
HAL Id: hal-01288900 \\ https://hal.science/hal-01288900
}

Submitted on 15 Mar 2016

HAL is a multi-disciplinary open access archive for the deposit and dissemination of scientific research documents, whether they are published or not. The documents may come from teaching and research institutions in France or abroad, or from public or private research centers.
L'archive ouverte pluridisciplinaire HAL, est destinée au dépôt et à la diffusion de documents scientifiques de niveau recherche, publiés ou non, émanant des établissements d'enseignement et de recherche français ou étrangers, des laboratoires publics ou privés. 


\title{
A solidification model for the columnar to equiaxed transition in welding of a Cr-Mo ferritic stainless steel with Ti as inoculant
}

\author{
V. Villaret, F. Deschaux-Beaume*, C. Bordreuil \\ Laboratoire de Mécanique et Génie Civil (LMGC), Université de Montpellier, CNRS, Montpellier, France
}

\begin{abstract}
A B S T R A C T
A two step modeling for predicting the grain morphology - columnar or equiaxed - of the melting zone in welding is proposed. The first step consists in numerically compute, using the Finite Element Method, the thermal fields around the weld pool boundary, according to the welding parameters. The second step consists in a post-treatment of the thermal field in order to compute the undercooling in the liquid all along the solidification front of the columnar grains, and finally to compute the fraction of equiaxed grains formed in the undercooling zone. The modeling takes into account the effect of the convection phenomena on the undercooling, and is based on an heterogeneous nucleation model specially adapted to steels inoculated with titanium. The nucleation model involves the size distribution of Ti-rich refractory compounds formed in the weld pool, that act as heterogeneous nucleation sites. A method to identify this distribution is also presented. The comparison of the modeling with experimental results of welding with a Gas Tungsten Arc Welding process has proved its ability to predict the columnar to equiaxed transition of a ferritic stainless steel inoculated with Ti.
\end{abstract}

Keywords: Solidification, Microstructure, Modeling, Ferritic stainless steel, Welding

\section{Introduction}

The welding remains the main joining process used for manufacturing structural metallic parts. Unfortunately, this joining process can have a detrimental effect on the material properties, specially in the melted zone, where both composition (when a filler wire is used) and microstructure are changed. In particular, the formation of a fully columnar grain structure in the melted zone during welding can be a major handicap, as it shown in a previous paper (Villaret et al., 2013a), because this type of structure has a very low strength. According to Flemings (1974), it is possible however, under certain conditions, to modify the morphology of the grains in the melted zone from a columnar to an equiaxed structure, as evidenced by Greer et al. (2003) in the case of aluminum alloys. This kind of structure significantly improves the ductility of the melted zones (Petersen, 1973) and decreases the transition temperature (Lakshminarayanan and Shanmugam, 2009), especially if, as evidenced by Bayraktar et al. (2006), the obtained equiaxed grain is fine. This transition from columnar grains to equiaxed grains, so called CET (Columnar to Equiaxed Transition) depends on the temperature field and its evolution in the solidification area. Two

\footnotetext{
* Corresponding author.

E-mail address: frederic.deschaux-beaume@umontpellier.fr (F. Deschaux-Beaume)
}

parameters have a particular importance on the solidification morphology, the thermal gradient at the solidification front $G$, and the moving velocity of this front $\mathrm{V}$. It is generally admitted (Kurz and Fisher, 1992) that a decrease in the G/V ratio promotes the CET.

The dominant theory for explaining the CET implies the nucleation and growth of new equiaxed grains in the undercooled liquid near the solidification front that stop the columnar grain growth, by mechanical interaction according to Kurz et al. (2001), or by solutal interaction according to Martorano et al. (2003). The cooling conditions promoting a high undercooling in the liquid near the solidification front, i.e., a low G/V ratio, favor the nucleation of new equiaxed grains and consequently the CET. In welding, an increase of the welding speed for instance can decrease the G/V ratio, thus promotes the equiaxed grain structure and, as evidenced by Bayraktar et al. (2006), improves the ductility of the melted zone. However, the number of nuclei giving equiaxed grains, formed in the undercooled liquid, is mainly controlled by the weld pool composition, and specially by the content of some elements favoring the formation of nucleation sites. According to Ostrowski and Langer (1979), titanium for instance has a very active role on the morphology of solidification grains for many alloys. In the case of ferritic stainless steels, it has been shown in a previous paper (Villaret et al., 2013b) that Ti forms refractory compounds in the liquid alloy that provide heterogeneous nucleation sites for equiaxed grains. The exact nature of these compounds is not always identified, and Villafuerte and Kerr (1990), Villafuerte et al. (1995) indicate 

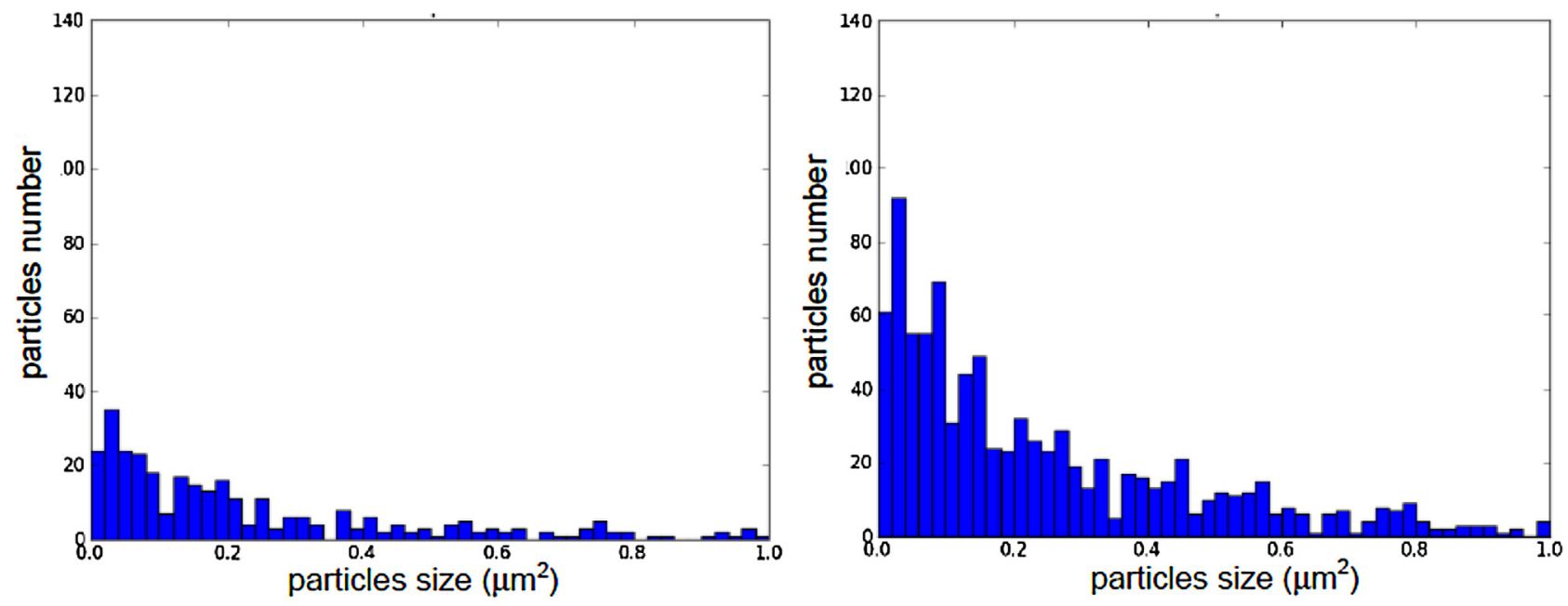

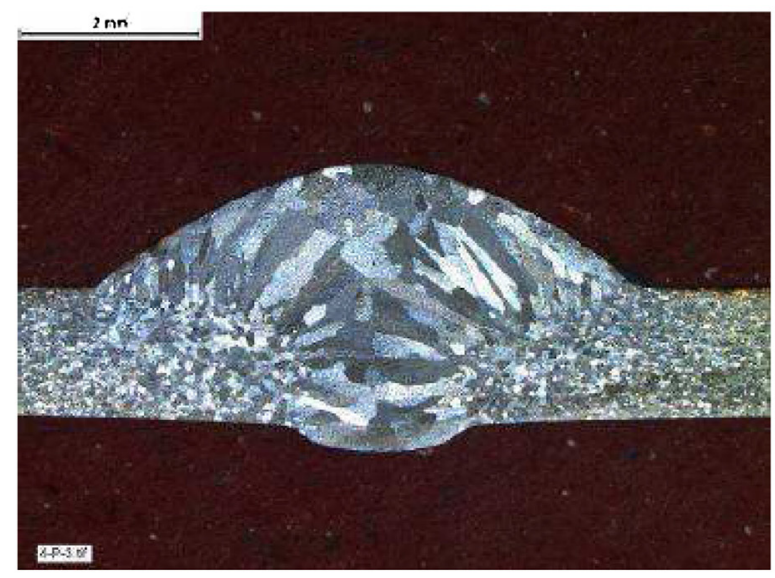

(a)

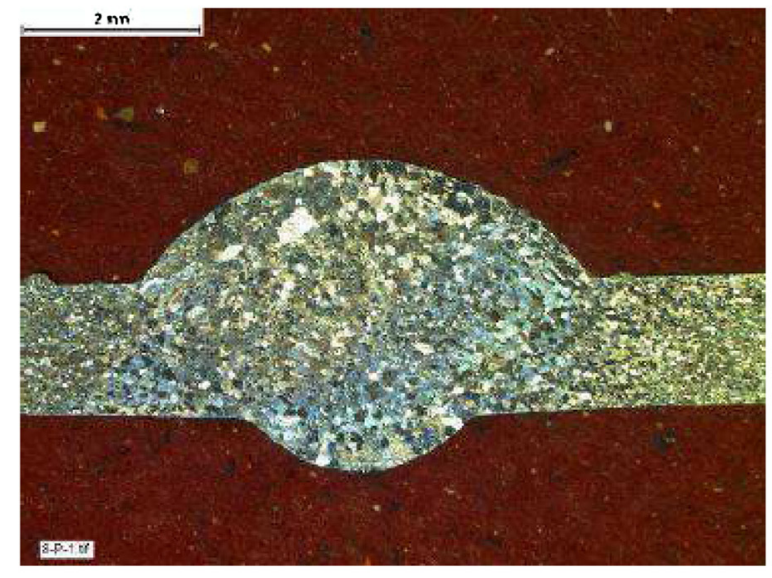

(b)

Fig. 1. Ti-rich precipitates size distribution and grain morphology in melting zones containing $0.07 \% \mathrm{Ti}$ (a), and $0.15 \% \mathrm{Ti}$ (b).

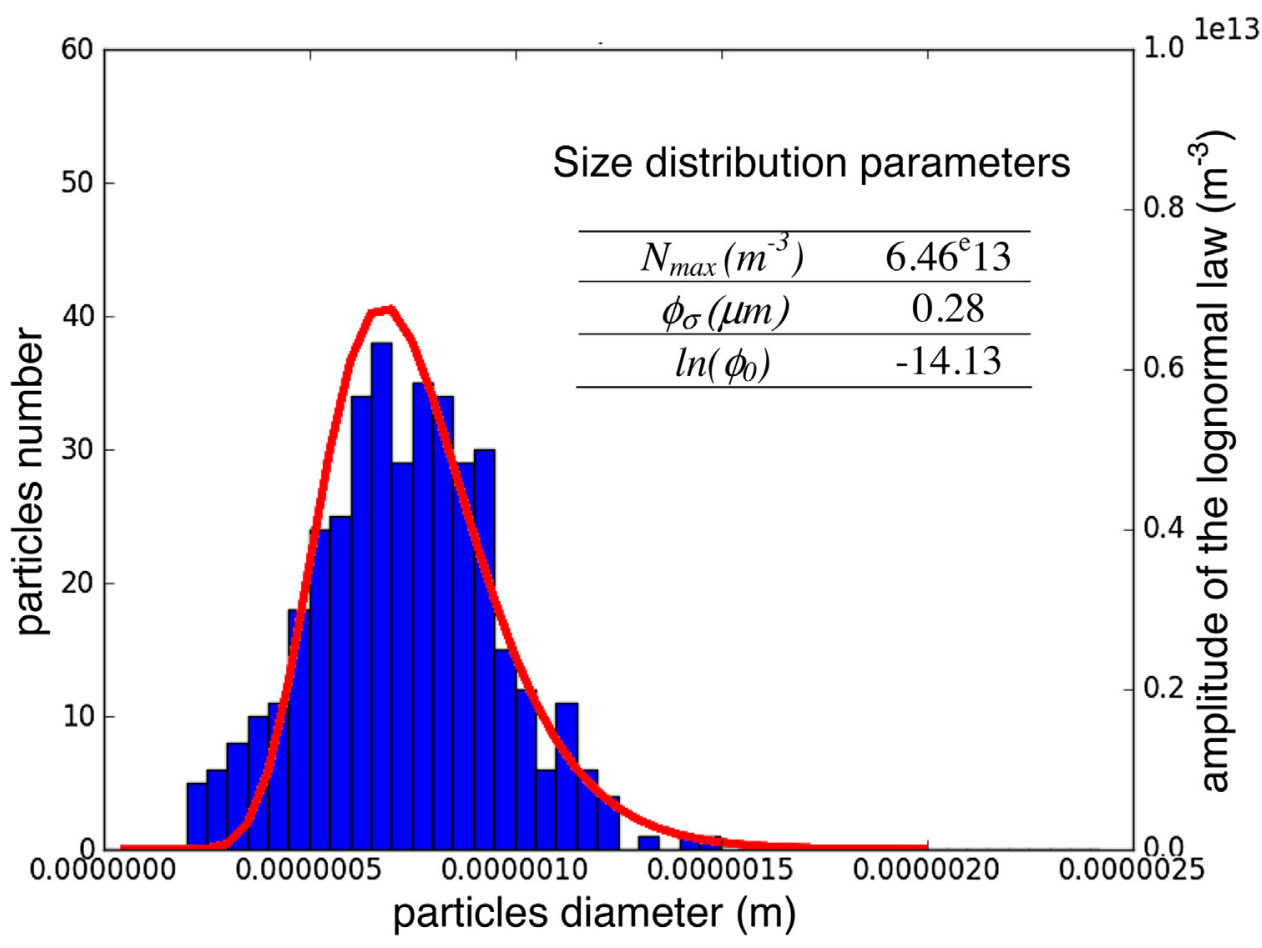

Fig. 2. Size distribution of Ti-rich particles in K44 steel melting zone, and distribution parameters of the associated lognormal law. 


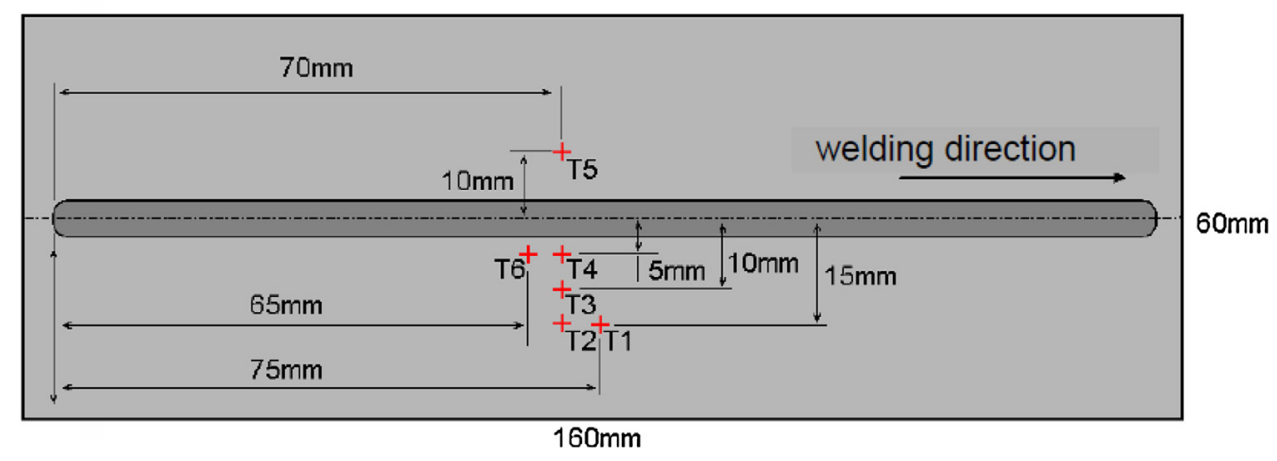

Fig. 3. Sample geometry for welding tests and thermocouples location (T1 to T6).

it seems to vary depending on the composition and cooling conditions.

As the formed grain structure in the melting zone has a primary importance on the weld properties, some modeling have been developed in order to try to predict the microstructure formed after welding. The modeling based on Cellular Automaton, generally coupled to Finite Element (CAFE) methods, recently developed, were applied by Rappaz et al. (1996) and Carozzani et al. (2012) to predict with a rather good accuracy the grain structure formed during ingot casting, or by Chiocca et al. (2015) for weld solidification, but needs very long computation time. At the opposite, the classical models based on Hunt's theory (Hunt, 1984) are more simple methods to predict if the CET occurs or not during the solidification, as demonstrated by Kurz et al. (2001), but require the preliminary determination of the equiaxed grain density, that is not predictable by the model, and that can change, as evidenced by Gaumann et al. (1997), according to the cooling rate.

In this paper, an alternative modeling for predicting solidification grain morphology in welding, based on a two step computation, is presented. The first step consists in a thermal numerical simulation of the welding process, in order to predict the thermal field evolution all around the solidification front. The second step consists in modeling, from this thermal field evolution, the grain solidification by the combination of the columnar growth model previously proposed by Niel et al. (2013), the nucleation model developed by Tronche et al. (2000), and a CET criteria derived from the Hunt's criteria (1984). The two steps of the mod- eling are first described, and then applied to predict the CET during Gas Tungsten Arc Welding of a ferritic stainless steel. The model predictions are compared to experimental results.

\section{Thermal modeling of welding}

A simplified thermal modeling of the welding process is retained to predict the thermal fields in the solidification area, using the commercial finite element software Sysweld. The welded sample is considered as a solid submitted to conduction heat transfer, with boundary conditions to model heat transfers between the sample and the surrounding environment. The heat source of the welding process is modeled by a heat flux distribution located on the surface of the sample.

\subsection{Geometry and mesh}

The modeled samples are thin sheets of $160 \times 60 \times 1.8 \mathrm{~mm}^{3}$ melted in their centerline using a Gas Tungsten Arc Welding (GTAW) process without filler metal. In order to reduce computation time, a two-dimensional simulation is chosen in the plane of the sample surface, as the melting zone is fully penetrated and have a rather similar width in back and upper side for a low sheet thickness. Due to the centerline symmetry, only half a sample is modeled and meshed with linear rectangular elements with 4 nodes. The elements around the symmetry axis, that are closed to the trajectory of the heat source center, suffer higher thermal gradients, and are

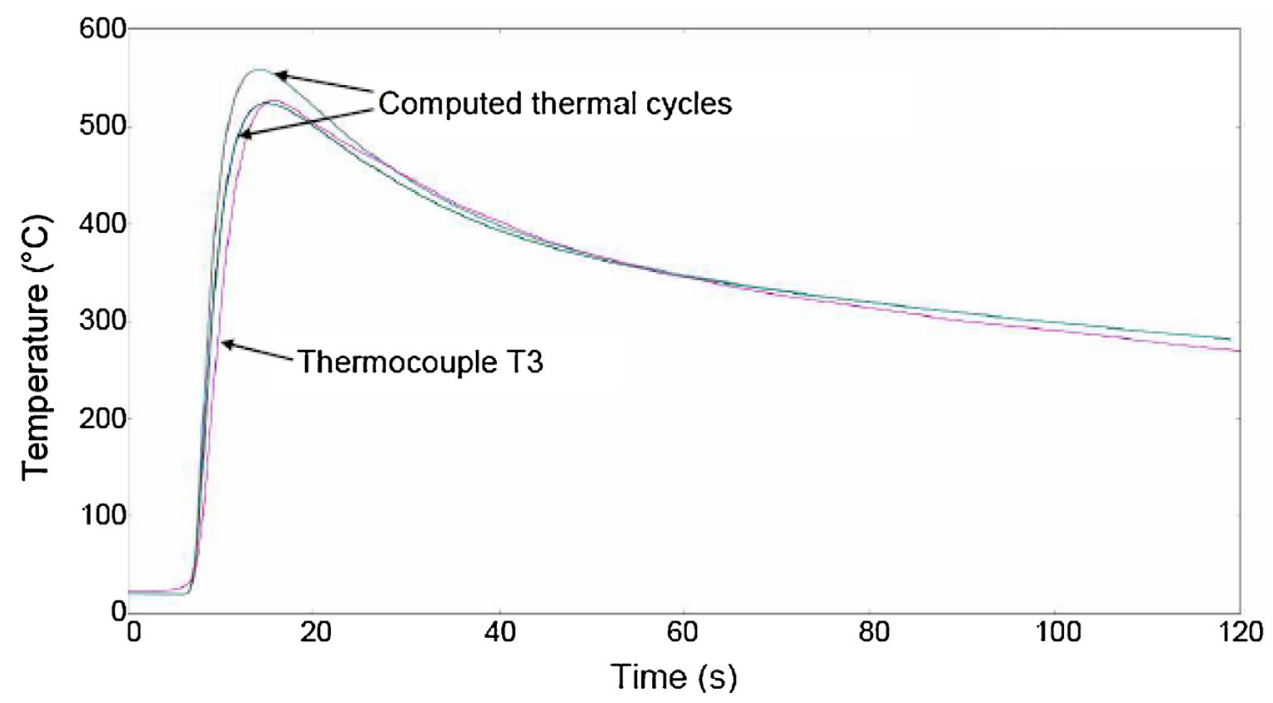

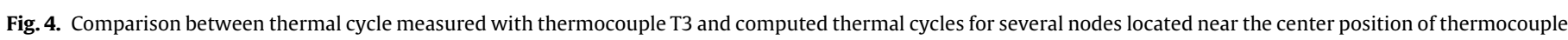
T3. 

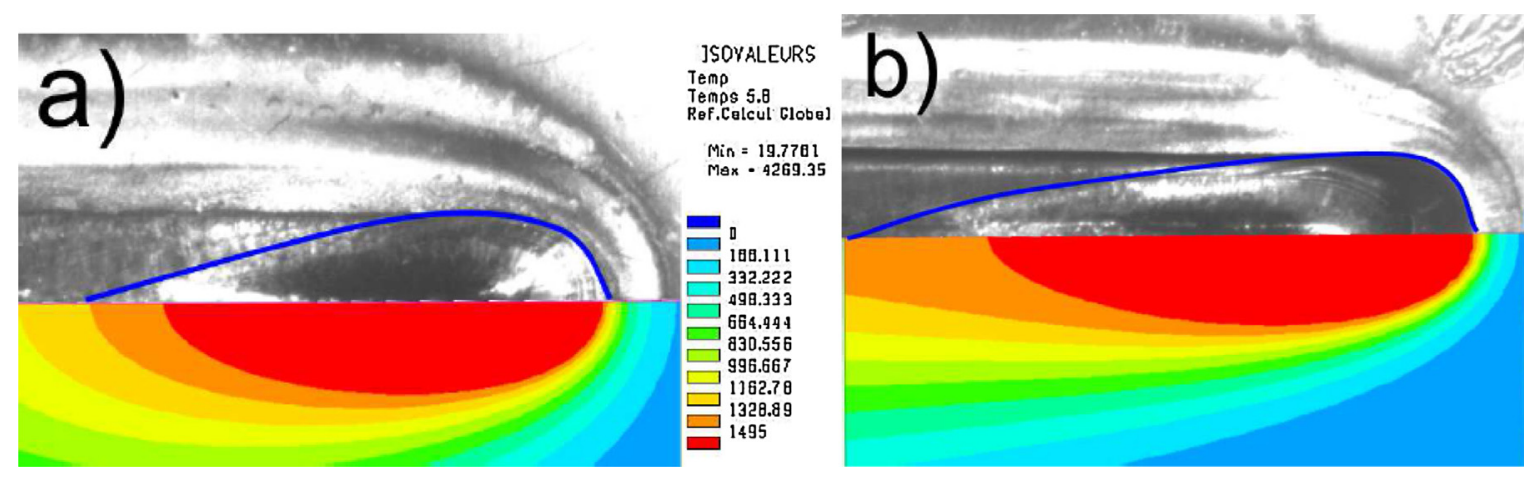

ISOVALEURS

Temp

Temps 3.4 Gef.Colcul Global

$\operatorname{Min}=19.8685$

$\operatorname{Max}=3477.59$

Fig. 5. Comparison between numerical and experimental weld pool boundaries for welding tests 1 (a) and 2 (b).

more finely meshed to get a better computation accuracy. The center of the heat source is positioned at the initial time on the left of the symmetry axis of the sample, and is translated along the y-axis at a speed corresponding to the welding speed.

\subsection{Heat equation}

Conduction heat transfers in solids are governed by the heat equation:

$\rho C p \cdot \frac{\partial T}{\partial t}-\operatorname{div}(\lambda \cdot \operatorname{grad} T)=Q(x, y, z, t)$

with $\mathrm{T}$ the temperature $(\mathrm{K}), \rho$ the density of the material $\left(\mathrm{kg} \mathrm{m}^{-3}\right)$, Cp the specific heat $\left(\mathrm{J} \mathrm{kg}^{-1} \mathrm{~K}^{-1}\right), \lambda$ the thermal conductivity $\left(\mathrm{W} \mathrm{m}^{-1} \mathrm{~K}^{-1}\right.$ ), and $\mathrm{Q}$ a potential source of external heat.

Solving this equation in any point of the sample in a Lagrangian $(\mathrm{O}, \mathrm{x}, \mathrm{y}, \mathrm{z})$ coordinate system associated to the sample, and at each time step, allows to know the evolution of the temperature field in the sample under the effect of the heat input $\mathrm{Q}$ corresponding to the welding source.

To take into account the latent heat of fusion $\mathrm{Lf}(\mathrm{Lf}=\mathrm{Hl}-\mathrm{Hs})$, the specific heat is increased within the solidification range (between Ts and $\mathrm{Tl}$ ) such as:

$\int_{T S}^{T l} C p d T=L f$

To take into account the increase in heat transfers in the weld pool due to convection phenomena, the thermal conductivity of the material is artificially increased from the liquidus temperature.

\subsection{Boundary and initial conditions}

The initial temperature of the specimen is taken equal to $20^{\circ} \mathrm{C}$ for all the nodes.

The heat transfers between the sample and the outer environment by convection and radiation are modeled by a flow imposed on all outer surfaces (Neumann condition):

$-\lambda \frac{\partial T}{\partial n}=h\left(T-T_{0}\right)+\varepsilon \sigma\left(T^{4}-T_{0}^{4}\right)$

with $\mathrm{n}$ the normal to the outer surface, $\mathrm{h}$ the convection exchange coefficient, $\varepsilon$ the emissivity of the material, and $\sigma$ the StephanBoltzmann constant.

\subsection{Heat source}

The heat source is modeled by a heat flow density imposed on the sample surface interacting with the arc. A Gaussian distribution is chosen, centered on the arc axis, moving in translation along the $y$-axis at a speed equal to the welding speed Vs. The surface distribution of heat flow density is then given by:

$Q(r)=\frac{2 Q_{0}}{\pi R_{s}} \exp \frac{-3 r^{2}}{R_{s}^{2}}$

with $\mathrm{Q}_{0}$ the Gaussian amplitude parameter, Rs the width parameter, and $r$ the distance to the center of the source, defined by:

$r=\left(X-X_{0}\right)^{2}+\left(Y-Y_{0}-V_{s} t\right)^{2}$

where $\mathrm{X}_{0}$ and $\mathrm{Y}_{0}$ are the coordinates of the initial position of the heat source, and the time.

The total power transmitted to the sample by this distribution, obtained by integrating the relationship (4) for $r$ values from 0 to infinity, must match the effective welding power. One can then (a)

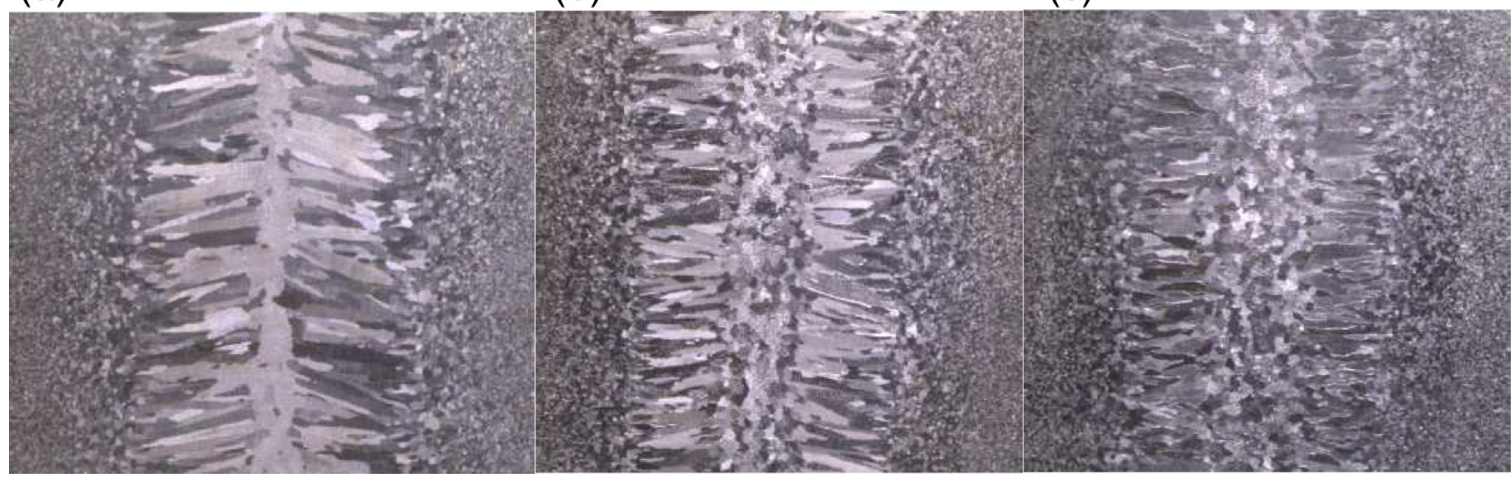

Fig. 6. Micrograph in top view of the melting zone corresponding to the welding test 1 (a), 2 (b) and 3 (c). 


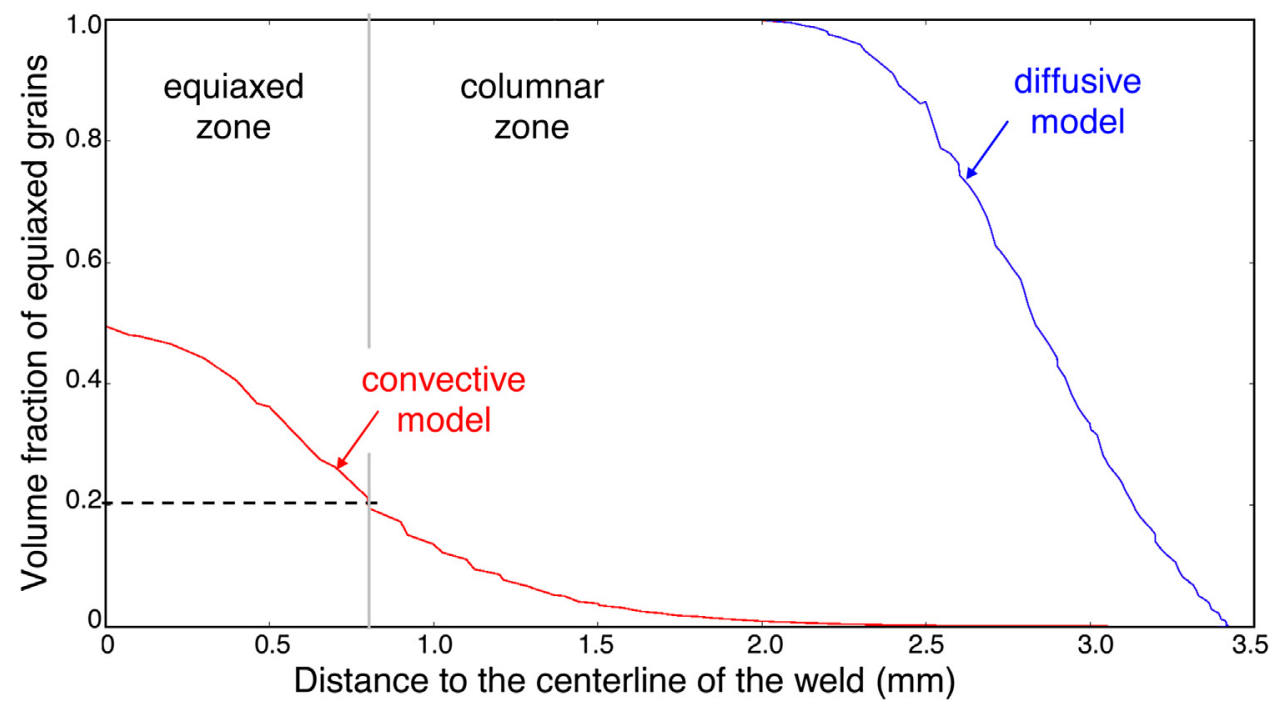

Fig. 7. Evolution of the volume fraction of equiaxed grains in the melting zone according to the distance to the centerline of the weld.

deduce the following relation between the distribution parameters and the process parameters:

$Q_{0}=\eta \cdot U I$

with $U$ the welding voltage, I the welding current and $\eta$ the process efficiency.

\section{Modeling of the CET}

The modeling is based on the approach developed by (Niel et al., 2013) coupled to the "athermal" nucleation model proposed by Greer (Tronche et al., 2000). It consists in compute the growth rate of columnar grains all around the solidification front, and the volume of nucleated equiaxed grains in the undercooled liquid near this front. When the volume fraction of equiaxed grains reaches a given limit at one point of the solidification front, then the CET occurs at this point.

\subsection{Growth rate of columnar grains}

The first step of the modeling consists in calculating the growth rate of columnar grains, all around the solidification front located on the rear border of the weld pool. It is supposed in this step the solidification front is on the liquidus isothermal line.

The growth direction of columnar grains is often supposed to be rather perpendicular to the liquidus isothermal line. However, experience indicates a divergence between heat flux direction and growth direction, specially for high welding speed, due to the preferential growth direction in crystalline materials.

In the modeling, the orientation of grains is given by the $\alpha$ angle, defined as the angle between the welding direction and the growth direction. The variation of this angle is calculated from eq. (7):

$\dot{\alpha}=A\left(\alpha-\alpha_{n}\right)$

where $\alpha \mathrm{n}$ is the angle between welding direction and heat flux, and A is a constant chosen for a given material according to preliminary welding results.

For each time step, the growth direction changes according to Eq. (8):

$\alpha(t+d t)=\alpha(t)+\dot{\alpha} d t$
The growth rate of columnar grains $\mathrm{V}$ is given by:

$V=V_{s} \frac{\cos \alpha_{n}}{\cos \left(\alpha-\alpha_{n}\right)}$

where Vs is the welding speed.

\subsection{Undercooling}

As suggested by Dantzig and Rappaz (2009), the curvature undercooling as well the undercooling associated with the attachment kinetics are neglected. The undercooling $\Delta \mathrm{T}_{\mathrm{S}}$ then results only on constitutional undercooling $\Delta \mathrm{T}_{\mathrm{C}}$ and thermal undercooling $\Delta \mathrm{T}_{\mathrm{T}}$ (Dantzig and Rappaz, 2009):

$\Delta T_{s}=\Delta T_{T}+\Delta T_{C}=\frac{L_{f}}{c_{p}} I v_{3 D}\left(P e_{T}\right)-m_{l} C_{0}\left(\frac{\left(1-k_{0}\right) I v_{3 D}\left(P e_{C}\right)}{1-\left(1-k_{0}\right) I v_{3 D}\left(P e_{C}\right)}\right)$

with $\mathrm{m}_{1}$ the liquidus slope, $\mathrm{C}_{0}$ the solute concentration in the liquid, $\mathrm{k}_{0}$ the partition coefficient of solute, and Iv3D the 3D Ivantsov function given by:

$I v_{3 D}\left(P e_{T}\right)=P e_{T} \exp \left(P e_{T}\right) \int_{P e_{T}}^{\infty} \frac{e^{-s}}{s} d s$

and $\mathrm{Pe}_{\mathrm{T}}$ and $\mathrm{Pe}_{\mathrm{C}}$ respectively the thermal and solutal Peclet numbers, given by:

$P e_{T}=\frac{V R_{\text {tip }}}{2 \alpha_{l}}$

$P e_{C}=\frac{V R_{\text {tip }}}{2 D_{l}}$

with $R_{\text {tip }}$ the dendrite tip radius, $\alpha_{1}$ the thermal diffusivity, and $D_{1}$ the diffusion coefficient of solute.

Supposing thermal and solutal Peclet numbers are below to 1, one can use the approximation of Ivantsov fonction proposed by Dantzig and Rappaz (2009) :

$I v_{3 D} P e \approx 1.5 P e^{0.8}$

The undercooling is then given according to the growth rate of columnar grains by the simplified expression:

$$
\begin{aligned}
& \Delta T_{s}=\Delta T_{T}+\Delta T_{c}=1.5 \pi^{0.8} \Gamma_{s l}^{0.4} \\
& \frac{L_{f} D_{l}^{0.8}-m_{l} C_{0} 1-k_{0} C_{p} \alpha_{l}^{0.8}}{C_{p}{ }^{0.6} \alpha_{l}{ }^{0.4} D_{l}^{0.4} L_{f} D_{l}-m_{l} C_{0} 1-k_{0} C_{p} \alpha_{l}{ }^{0.4}} V^{0.4}
\end{aligned}
$$

with $\Gamma_{\mathrm{sl}}$ the Gibbs Thomson coefficient. 


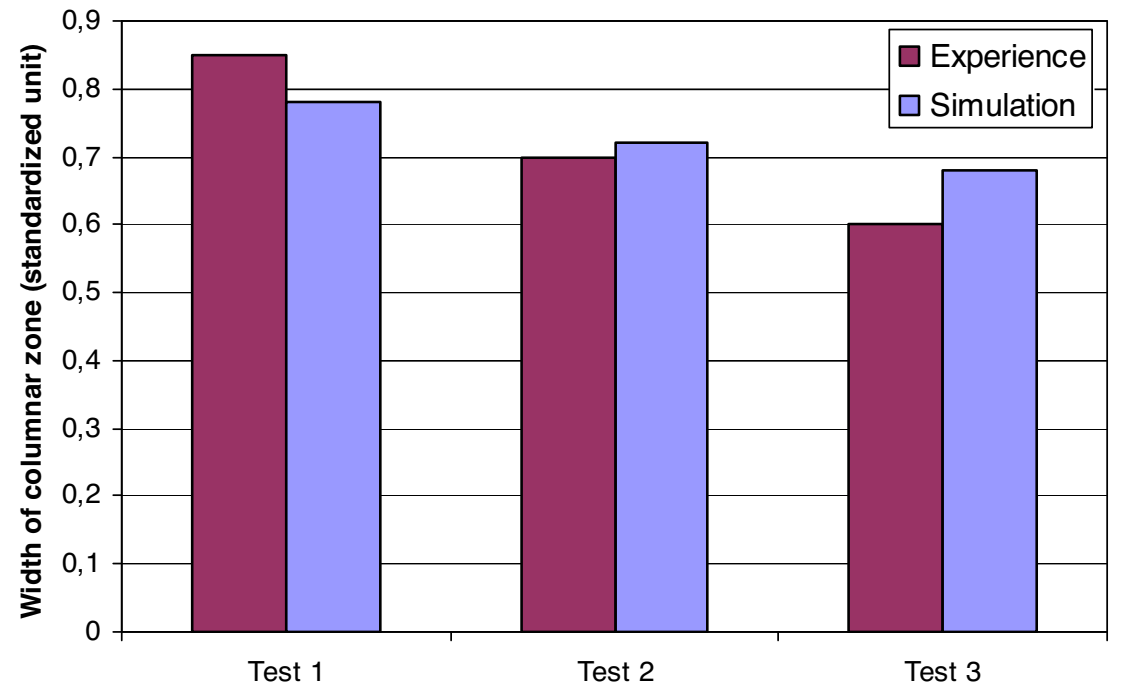

Fig. 8. Comparison of the width of the columnar grains zone computed and experimentally observed for the three welding tests.

3.3. Nucleation and growth of equiaxed grains in the undercooling region

The width $\delta$ of the undercooling region near the columnar solidification front, supposed very small, is calculated from the undercooling $\Delta T_{S}$ and the maximal thermal gradient $\mathrm{G}$ (normal to the isothermal surface) on each point of the liquidus. Supposing a linear temperature variation in this area, the undercooling width $\delta$ is given by:

$\delta=\frac{\Delta T_{S}}{G}$

The nucleation of equiaxed grains is supposed to be possible only in this area. The undercooling on each point of this zone then varies between $\Delta$ Ts at the columnar front to 0 at the distance $\delta$ from this front.

\subsection{Number of formed nuclei}

The athermal nucleation model developed by Greer to predict solidification during casting of aluminum alloys (Tronche et al., 2000) has been retained. This model is well suited for heterogeneous nucleation from small solid particles (Greer et al., 2003), as in ferritic stainless steels inoculated with $\mathrm{Ti}$, in which ferritic grains nucleate from Ti-rich refractory compounds formed in the weld pool (Villaret et al., 2013b).

It is supposed the size distribution of Ti-rich precipitates can be approximated using a lognormal distribution:

$\frac{d n}{d \phi}=\frac{n_{\max }}{\phi_{\sigma} \sqrt{2 \pi}} \frac{1}{\phi} \exp \left(-\frac{1}{2}\left(\frac{\ln \phi-\ln \phi_{0}}{\phi_{\sigma}}\right)^{2}\right)$

with $\Phi$ the precipitate diameter, $n$ max the precipitates number density, $\Phi_{\sigma}$ and $\ln \Phi_{0}$ the distribution parameters, corresponding respectively to the standard deviation and the mean.

According the Greer nucleation model, each precipitate of diameter $\varphi$ is associated to a nucleation undercooling $\Delta \mathrm{T}$ given by relation (18):

$\Delta T=\frac{4 \Gamma_{s l}}{\phi}$
One can deduce the nucleation undercooling distribution, i.e. the number of nuclei "activated" according to the undercooling $\Delta \mathrm{T}$ :

$\frac{d n}{d \Delta T}=\frac{n_{\max }}{\Delta T_{\sigma} \sqrt{2 \pi}} \frac{1}{\Delta T} \exp \left(-\frac{1}{2}\left(\frac{\ln \Delta T-\ln \Delta T_{0}}{\Delta T_{\sigma}}\right)^{2}\right)$

with $\Delta T_{\sigma}=\Phi_{\sigma}$ and $\Delta T_{0}=4 \Gamma_{\text {sl }} / \Phi_{0}$.

The number of nuclei formed in each elementary volume of the undercooling zone, associated to the undercooling $\Delta \mathrm{Ti}$ (between 0 and $\Delta \mathrm{Ts}$ ), is then given by relation (20):

$d n\left(\Delta T_{i}\right)=\frac{n_{\max }}{\Delta T_{\sigma} \sqrt{2 \pi}} \frac{1}{\Delta T_{i}} \exp \left(-\frac{1}{2}\left(\frac{\ln \Delta T_{i}-\ln \Delta T_{0}}{\Delta T_{\sigma}}\right)^{2}\right) d \Delta T_{i}(20)$

\subsection{Growth of equiaxed grains}

Once nuclei are formed, equiaxed grains will grow at a growth rate depending on the undercooling $\Delta T_{i}$ in the surrounding liquid volume, supposed to be at the same temperature than the nuclei. The growth rate of equiaxed grains $v_{e}$ follows the same growth law than columnar grains:

$v_{e}=A \Delta T_{i}^{2.5}$

with $A=\left(\frac{C_{p}^{0.6} \alpha_{l}^{0.4} D_{l}^{0.4}\left(L_{f} D_{l}-m_{l} C_{0}\left(1-k_{0}\right) C_{p} \alpha_{l}\right)^{0.4}}{1,5 \pi^{0.8} \Gamma_{s l}^{0.4}\left(L_{f} D_{l}^{0.8}-m_{l} C_{0}\left(1-k_{0}\right) C_{p} \alpha_{l}^{0.8}\right)}\right)^{2.5}$

During the time between the moment where the nuclei is formed, at time $t_{i}$, and the moment $t_{s}$ where the columnar front arrives (at the speed $V$ ), the equiaxed grain reaches a radius $R$ given by:

$R=\int_{t_{i}}^{t_{s}} v_{e} d t=\frac{A}{G V} \int_{\Delta T_{i}}^{\Delta T_{s}} \Delta T^{2.5} d \Delta T=\frac{A}{3.5 G V} \Delta T_{s}^{3.5}-\Delta T_{i}^{3.5}$

The volume of equiaxed grains formed at the undercooling $\Delta \mathrm{Ti}$ is then equal to:

$$
\begin{aligned}
& d V\left(\Delta T_{i}\right)=\frac{n_{\max }}{\Delta T_{\sigma} \sqrt{2}} \frac{1}{\Delta T_{i}} \exp -\frac{1}{2} \frac{\ln \Delta T_{i}-\ln \Delta T_{0}}{\Delta T_{\sigma}}{ }^{2} \\
& \frac{4}{3} \pi \frac{A}{3.5 G V} \Delta T_{s}^{3.5}-\Delta T_{i}^{3.5}{ }^{3} d \Delta T_{i}
\end{aligned}
$$


Table 1

Composition of the ferritic stainless steel K44 (Arcelor Mittal).

\begin{tabular}{lllllllllll}
\hline Element & $\mathrm{C}$ & $\mathrm{Si}$ & $\mathrm{Mn}$ & $\mathrm{Cr}$ & $\mathrm{Mo}$ & $\mathrm{Al}$ & $\mathrm{Nb}$ & $\mathrm{Ti}$ & $\mathrm{V}$ & $\mathrm{Fe}$ \\
\hline Weight\% & 0.02 & 0.45 & 0.39 & 18 & 2.09 & 0.007 & 0.29 & 0.16 & 0.1 & 79.9 \\
\hline
\end{tabular}

Table 2

Material properties used for thermal numerical simulation.

\begin{tabular}{rlll}
\hline $\begin{array}{l}\text { Temperature } \\
\left({ }^{\circ} \mathrm{C}\right)\end{array}$ & $\begin{array}{l}\text { Specific heat } \mathrm{Cp} \\
\left(\mathrm{J} \mathrm{kg}^{-1} \mathrm{~K}^{-1}\right)\end{array}$ & $\begin{array}{l}\text { Thermal Conductivity } \lambda \\
\left(\mathrm{W} \mathrm{m}^{-1} \mathrm{~K}^{-1}\right)\end{array}$ & $\begin{array}{l}\text { Density } \rho \\
\left(\mathrm{kg} \mathrm{m}^{-3}\right)\end{array}$ \\
\hline 23 & 452 & 19.7 & 7675 \\
200 & 522 & 21.0 & 7632 \\
400 & 608 & 21.9 & 7581 \\
600 & 840 & 22.8 & 7526 \\
800 & 612 & 23.0 & 7467 \\
1000 & - & - & 7392 \\
\hline
\end{tabular}

The total volume of equiaxed grains is equal to the sum of the volumes of grains formed at all undercooling between 0 and $\Delta \mathrm{T}_{\mathrm{S}}$ :

$V_{g e}\left(\Delta T_{s}\right)=\int_{0}^{\Delta T_{s}} d V\left(\Delta T_{i}\right)$

To take into account the decrease of the growth rate due to the possible blocking between adjacent grains, one considers the volume fraction of equiaxed grains follows an Avrami law:

$f_{g e}=1-\exp \left(-V_{g e}\left(\Delta T_{s}\right)\right)$

\section{Identification of data required for computation}

The modeling described in the previous sections has been employed to study the behavior during GTAW of the melted zone of a ferritic stainless steel containing $\mathrm{Ti}$ as inoculant. Previous experimental results validated the assumption of an heterogeneous nucleation mechanism promoting the CET during Gas Metal Arc Welding of such stainless steels, and then confirms the choice of a nucleation theory based on the Greer model. In a previous paper (Villaret et al., 2013b), it has been shown the CET is favored by a high Ti content in the filler metal (upper to $0.15 \mathrm{wt} \%$ ), that forms refractory compounds in the weld pool acting as heterogeneous nucleation sites. These refractory compounds can be detected by Scanning Electron Microscopy (SEM) and Energy Dispersive Spectroscopy (EDS) analyses, and their distribution are directly correlated to the Ti content. However the CET, that occurs when the columnar growth is stopped by the equiaxed grains formed in the undercooling zone, is controlled for a given composition (i.e., for a given content of inoculants as $\mathrm{Ti}$ ), by thermal conditions during solidification, that determine the undercooling. In order to test the ability of the proposed modeling to predict the CET, a stainless steel having a controlled $\mathrm{Ti}$ content of $0.16 \mathrm{wt} \%$, namely the K44 alloy supplied by Arcelor Mittal (Table 1), is used.

\subsection{Thermal simulation}

The physical characteristics of the material $(\rho, \mathrm{Cp}, \lambda)$ vary with temperature, and are given in Table 2 up to 800 or $1000^{\circ} \mathrm{C}$. These characteristics are assumed to be constant beyond these temperatures up to the solidus temperature of the material.

The heat source parameters are chosen using literature data and experimental considerations. The width parameter Rs is chosen to be rather similar to the arc radius, and de process efficiency $\eta$ is chosen according to literature data and validated by comparison between preliminary simulations and previous welding tests results (Table 3 ).
Table 3

Heat source parameters and heat transfer coefficients used for thermal simulation.

\begin{tabular}{llll} 
Rs $(\mathrm{mm})$ & Process efficiency $\eta$ & Emissivity $\varepsilon$ & Convection coefficient $h\left(\mathrm{~W} / \mathrm{m}^{2}\right)$ \\
\hline 2 & 0.5 & 0.05 & 7.5
\end{tabular}

Radiation and convection heat transfer coefficients are chosen according to previous instrumented welding tests, using comparisons between numerical and experimental cooling rates (Table 3).

\subsection{CET modeling}

\subsubsection{Physical properties of the material}

The physical properties required for the computation are gathered in Table 4. Formulations expressing the relationship between undercooling and growth rate (15) are given for binary alloys. In the case of multi-component alloys, one can consider an equivalent pseudo-system. $\mathrm{m}_{1}, \mathrm{C}_{0}$, and $\mathrm{k}_{0}$ parameters are then replaced by the following expressions (Mingbo Yang and Fusheng Pan, 2009):

$C_{0}=\sum_{i} C_{i} \quad m_{l}=\sum_{i} \frac{m_{i} C_{i}}{C_{0}} \quad k_{0}=\sum_{i} \frac{m_{i} C_{i} k_{i}}{m_{l} C_{0}}$

where $C_{i}$ is the concentration of element $i, m_{i}$ and $k_{i}$ respectively the liquidus slope and the partition coefficient in the binary diagram Fe-element $i$.

Only $\mathrm{Cr}$ and Mo elements are took into account, that have the higher contents in the material, to compute these parameters.

\subsection{Taking account of convection in the weld pool}

Using values of Table 4, the undercooling at the columnar solidification front, composed of dendrite tips, given by eq. 15 , becomes:

$\Delta T_{s}=105.3 V^{0.4}$

with $\mathrm{V}$ in $\mathrm{m} / \mathrm{s}$.

Eq. (15) is established using a "marginal stability criteria" that suppose the dendrite tip radius Rtip is equal to the minimal wavelength of disturbance $\lambda$ min producing the instability of a planar solidification front (Dantzig and Rappaz, 2009). According to this criteria, the dendrite tip radius is given by:

$R_{\text {tip }}=\lambda_{\text {min }}=2 \pi \sqrt{\frac{\Gamma_{s l}}{m_{l} G_{c}-G}}$

where $G c$ and $G$ are respectively the solute gradient and the thermal gradient in the liquid at the solidification front, given, according to the Ivantsov analyze (Dantzig and Rappaz, 2009), by:

$G=-\frac{2 P e_{T} L_{f}}{C p R_{\text {tip }}}$

$G_{C}=-\frac{2 P e_{C} C_{l}\left(1-k_{0}\right)}{R_{\text {tip }}}$

The dendrite tip radius calculated with the values of Table 4, of about $0.5 \mu \mathrm{m}$, is very low compared to that generally observed after welding of these ferritic stainless steel, which is about $10 \mu \mathrm{m}$. That seems to prove the convection phenomena due to fluid flow acting in the weld pool have a significant effect on the solidification mechanism, by promoting the thermal and solute transfers, and then by increasing the thermal and solute limit boundary layers, given by $\delta_{T}=2 \alpha_{l} / V$ and $\delta_{C}=2 D_{l} / V$ for pure diffusive mechanisms. If one considers thermal and solute boundary layers have the same thickness due to the convection, the effect of fluid flow can be artificially traduced by an increase of the thermal and solute diffusivity coefficients. The "equivalent diffusivity" Deq corresponding to a dendrite tip radius of $10 \mu \mathrm{m}$ is $2.310^{-5} \mathrm{~m}^{2} \mathrm{~s}^{-1}$ for a moving velocity of the 
Table 4

Physical properties used for the solidification modeling.

\begin{tabular}{|c|c|c|}
\hline Physical property & Value & References \\
\hline $\mathrm{D}_{\mathrm{l}}$ (diffusion coefficient of solute into the liquid) & $3.10^{-9} \mathrm{~m}^{2} \mathrm{~s}^{-1}$ & Dantzig and Rappaz(2009) \\
\hline $\mathrm{m}_{\mathrm{l}}$ (liquidus slope) & -186 & Kagawa and Okamoto(1984) \\
\hline $\mathrm{k}_{0}$ (partition coefficient of solute $\mathrm{ms} / \mathrm{ml}$ ) & 0.61 & Kagawa and Okamoto (1984) \\
\hline$C_{0}$ (solute concentration in the liquid) & 0.209 & Kagawa and Okamoto (1984) \\
\hline$\Gamma_{\mathrm{sl}}$ (Gibbs Thomson coefficient, $\gamma \mathrm{sl} / \rho \Delta \mathrm{S}_{\mathrm{f}}$ ) & $1.910^{-7} \mathrm{~m} \mathrm{~K}$ & Dantzig and Rappaz (2009) \\
\hline$\gamma_{\mathrm{sl}}$ (solid-liquid surface tension) & $0.204 \mathrm{~J} \mathrm{~m}^{-2}$ & Dantzig and Rappaz (2009) \\
\hline$\rho \Delta S_{\mathrm{f}}$ (volume entropy of fusion) & $1.0710^{6} \mathrm{~J} \mathrm{~m}^{-3} \mathrm{~K}^{-1}$ & Dantzig and Rappaz (2009) \\
\hline$\alpha \mathrm{l}$ (thermal diffusivity in liquid steel) & $6.10^{-6} \mathrm{~m}^{2} \mathrm{~s}^{-1}$ & Nishi et al. (2003) \\
\hline $\mathrm{Cp}$ (specific heat) & $820 \mathrm{~J} \mathrm{~kg}^{-1} \mathrm{~K}^{-1}$ & Dantzig and Rappaz (2009) \\
\hline $\mathrm{L}_{\mathrm{f}}$ (latent heat of fusion) & $2.7610^{5} \mathrm{~J} \mathrm{~kg}^{-1}$ & Dantzig and Rappaz (2009) \\
\hline
\end{tabular}

solidification front of $0.005 \mathrm{~ms}^{-1}$, corresponding to a representative value in welding. By replacing $\alpha_{1}$ and $D_{1}$ by this new coefficient, Eqs. (15) and (27) become:

$$
\begin{aligned}
& \Delta T_{s}=\Delta T_{T}+\Delta T_{c}=1.5 \pi^{0.8} \\
& \frac{\Gamma_{s l}^{0.4}}{C_{p}^{0.6} D_{e q}^{0.4}}\left(L_{f}-m_{l} C_{0}\left(1-k_{0}\right) C_{p}\right)^{0.6} V^{0.4}=18.55 V^{0.4}
\end{aligned}
$$

with $\mathrm{V}$ in $\mathrm{m} / \mathrm{s}$.

\subsection{Precipitates distribution in the melting zone}

The nucleation model defines in Eq. (17) a precipitates distribution allowing the heterogeneous nucleation of equiaxed grains in the undercooling zone. In ferritic stainless steel, these precipitates are supposed to be titanium-based refractory compounds (Villaret et al., 2013b). One of the limitation of this model is it requires the identification of this precipitates distribution. In order to study this distribution, a methodology has been developed and tested on several stainless steels. The precipitates distribution analysis is carried out using a SEM equipped with INCA analysis setup developed by OXFORD ${ }^{\odot}$ (www.oxford-instruments.com). This tool allows to automatically detect and analyze by EDS, in a predefined field of the sample, particles using the grey level differences compared to the matrix. This tool gives for each detected particle its position, size, grey level and composition. The magnification of SEM images for the analyze is fixed at 700 to detect particles of less than $0.05 \mu \mathrm{m}$ in diameter. The analysis field consists in 9 SEM images representing a total surface area of about $0.5 \mathrm{~mm}^{2}$. A threshold of grey level is chosen to separate Ti-rich particles, that appear in dark grey on SEM images, and the matrix. The main elements of the steel, namely chromium and iron, plus $\mathrm{Ti}$ and $\mathrm{Nb}$ additive elements are analyzed by EDS on each detected particle, in order to confirm it is Ti-rich particle. That allows to eliminate from the distribution for instance porosities, that appears also in dark contrast in the SEM images. Finally, a dedicated Python routine is developed to select, according to the EDS analyses, the Ti-reach particles, and to compute their size distribution.

This analysis method has been used to compare the Ti-rich precipitates distribution of the melting zone of ferritic stainless steels containing various Ti contents, and revealed significant changes between the compositions. Fig. 1 shows the differences between 2 steels containing various Ti contents, and then various Ti-rich particles distributions. The first one, containing $0.07 \mathrm{wt} \%$ titanium, exhibit a columnar grain structure in the melting zone, except in the center-part and the top, where some coarse equiaxed grains are formed. Conversely, the second one, containing $0.15 \%$ titanium, exhibit a quasi-complete equiaxed grains structure in the melting zone. As a consequence of a higher Ti content, a greater number of Ti-rich precipitates is formed, and CET is promoted in the melting zone.
Table 5

Welding parameters for GTAW tests.

\begin{tabular}{lllc}
\hline & Voltage $(\mathrm{V})$ & Curent $(\mathrm{A})$ & Welding speed $(\mathrm{mm} / \mathrm{s})$ \\
\hline Test 1 & 13 & 155 & 4.9 \\
Test 2 & 13.5 & 253.5 & 9.5 \\
Test 3 & 14.5 & 303.5 & 12.9 \\
\hline
\end{tabular}

On the other hand, when a given composition is melted by GTAW process with various welding parameters, there is only minor changes in the precipitates distribution, that shows this distribution is mainly controlled by the steel composition. It is an interesting result because that means the precipitates distribution for a given steel composition has to be characterized only one time, whatever the welding parameters used.

Fig. 2 shows the size distribution of Ti-rich precipitates obtained by analyzing the melting zone of the K44 steel, containing $0.16 \%$ titanium, and the associated lognormal law. The parameters of the lognormal law are computed according to relations:

$\ln \phi_{0}=\sum_{i=1}^{n} \frac{n_{i}}{n_{T}} \ln \phi_{i}$

$\phi_{\sigma}=\sqrt{\sum_{i=1}^{n}\left(\ln \phi_{i}-\ln \phi_{0}\right)^{2} \frac{n_{i}}{n_{T}}}$

$n_{\max }=\sum_{i=1}^{n} \frac{n_{i}}{\Delta \phi}$

with $\mathrm{n}_{\mathrm{i}}$ the number of particles per volume unit of diameter $\Phi_{\mathrm{i}}, \mathrm{n}_{\mathrm{T}}$ the total number of particles per volume unit $\left(\mathrm{m}^{-3}\right)$, and $\Delta \Phi$ the width of the diameter classes.

The size distribution parameters identified from this analyze are given in Fig. 2. One can deduced from these parameters the distribution parameters of the "nucleation undercooling" distribution of Eq. (19).

\section{Comparison with experimental results}

In order to test the ability of the proposed modeling to predict the CET, bead on plate tests using GTAW process on K44 steel are carried out and analyzed for comparison to the simulation predictions.

\subsection{Experimental details}

Three GTAW welding tests have been achieved with various welding parameters chosen in order to produce increasing undercooling, and then to promote CET (Table 5). The welding speed and current have been simultaneously increased to obtain for each test a rather constant width of melting zone. A GTAW source Sincosalt AC/DC 400A, associated with an automatic water-cooled torch 
MEC4SAF-FRO equipped with a thoriated tungsten electrode (W$2 \% \mathrm{Th}$ ) of $2.4 \mathrm{~mm}$ diameter with a filing angle of $45^{\circ}$, has been used. The shielding gas is a $99.5 \%$ pure argon (ARCAL 1 Air Liquide) with a flow rate of $15 \mathrm{l} / \mathrm{min}$.

The samples are stainless steel sheets of $160 \times 60 \times 1.8 \mathrm{~mm}^{3}$. Six K-type sheathed thermocouples of $0.5 \mathrm{~mm}$ diameter were placed on each test specimen (Fig. 3), on the back face in order to protect them against the arc radiation, that can distort measurements. To keep thermocouples in position, they have been embedded in $0.5 \mathrm{~mm}$ diameter holes of $0.5 \mathrm{~mm}$ depth machined using hemispherical machining tools to improve contact surface between the sample and the thermocouples. Thermal acquisitions are made at a frequency of $20 \mathrm{~Hz}$ for $120 \mathrm{~s}$.

\subsection{Thermal modeling}

Fig. 4 compares the thermal cycle measured by thermocouple T3 with those computed by numerical simulation at the nodes located near the location of thermocouple T3. The center position of the thermocouple does not correspond exactly to a node position, so several thermal cycles corresponding to neighboring nodes are shown. Comparison between simulated and experimental temperature curves shows good agreement, excepts for the thermocouples closest to the melting zone (T4 and T6), where the computed maximum temperature is about $200^{\circ}$ higher than that measured. This can be explained in particular by the thermal inertia of the sheathed thermocouples and the imperfect contact with the sample.

Fig. 5 compares the computed isothermal lines corresponding to the liquidus temperature of the alloy with the boundary of the weld pool observed at the end of the fusion line. One can see that despite a comparable width, the length of the experimental weld pool is always greater than the simulated one. That can be due to the fact that the physical phenomena related to the fluid flow in the weld pool are not taken into account by the simulation. Note also that in the numerical simulation, we consider the boundary of the weld pool is given by the liquidus isotherm, while the real boundary at the rear of the weld pool may be at lower temperature due to the undercooling, and may not correspond to an isothermal surface. However, despite the simplification adopted in the thermal modeling, the weld pool boundary and the thermal field are globally well predicted for each welding condition.

\subsection{Prediction of the CET}

Fig. 6 shows in top view the grain structure of the melting zone formed for the three welding tests. One can see the fraction of equiaxed grains in the center part of the melting zone increases with the welding speed, as expected.

Fig. 7 shows the evolution of the equiaxed grain fraction in the melting zone computed from the thermal simulation of test 1, using the "diffusive model" (Eq. (27)) or the "convective model" (Eq. (31)) for undercooling computation. The content of equiaxed grains increases from the fusion line boundary to the centerline. One can see that if the correction proposed for taking into account the effects of convection phenomena is not applied, the fraction of equiaxed grains reaches high values, traducing a CET that promote the formation of equiaxed grains in the major part of the melting zone, what is not in agreement with the experimental results. The model taking into account the convection phenomena seems then more realistic. Comparing this first numerical result with experiment, the fraction of equiaxed grains producing the CET is chosen equal to $20 \%$. One can then compare the width of the columnar grain zone computed for the three welding tests with experiment. Fig. 8 shows the general tendency, that involves an increase of the width of the equiaxed grains zone with the increase of the welding speed, is well predicted by the modeling, despite a overestimation of the width of the columnar grain zone obtained with the highest welding speed.

\section{Conclusions}

- A modeling has been proposed to predict the columnar to equiaxed transition in the weld pool of ferritic stainless steels inoculated with $\mathrm{Ti}$, according to welding parameters.

- The first step of the modeling consists to a thermal numerical simulation of the welding process from which thermal fields all around the weld pool boundary are extracted.

- In a second step, the thermal data are post-treated in order to compute the undercolling, taking into account the effect of the convection phenomena, and the fraction of equiaxed grains formed in the undercooling zone all around the weld pool boundary.

- The modeling requires to know the size distribution of Ti-rich refractory compounds in the weld pool promoting the heterogeneous nucleation of equiaxed grains, that have been determined using a specific method based on SEM and EDS analyzes.

- The confrontation of the model to experimental results shows it can reproduce in a realistic manner the columnar and equiaxed zone in a ferritic stainless steel containing $\mathrm{Ti}$ as inoculant.

\section{Acknowledgements}

The authors wish to thank Air Liquide, Saint-Ouen l'Aumône, France for its financial support for this study, and Bertrand Petit, Laurent Faivre and Pierre-Olivier Santacreu, from Arcelor Mittal, Isbergue, France, for supplying the K44 steel.

\section{References}

Bayraktar, E., Moiron, J., Kaplan, D., 2006. Effect of welding conditions on the formability characteristics of thin sheet steels: mechanical and metallurgical effects. J. Mater. Process. Technol. 175, 20-26.

Carozzani, T., Digonnet, H., Bellet, M., Gandin, C.A., 2012. 3D CAFE simulation of a macrosegregation benchmark experiment. IOP Conf. Ser.: Mater. Sci. Eng. 33, 012087

Chiocca, A., Soulié, F., Bordreuil, C., Deschaux-Beaume, F., 2015. Microstructure generation during CuNi bead on plate: comparison of experimental results and CA prediction. 11th International Seminar Numerical Analysis of Weldability.

Dantzig, J.A., Rappaz, M., 2009. Solidification. EPFL Press, pp. 318-323.

Flemings, M.C., 1974. Solidification processing. Metall. Trans. 5, 2121-2134.

Gaumann, M., Trivedi, R., Kurz, W., 1997. Nucleation ahead of the advancing interface in directional solidification. Mater. Sci. Eng. A 226, 763-769.

Greer, A.L., Cooper, P.S., Meredith, M.W., Schneider, W., Schumacher, P., Spittle, J.A., Tronche, A., 2003. Grain refinement of aluminium alloys by inoculation. Adv. Eng. Mater. 5, 81-91.

Hunt, J.D., 1984. Steady state columnar and equiaxed growth of dendrites and eutectic. Mater. Sci. Eng. 65, 75-83.

Kagawa, A., Okamoto, T., 1984. Coefficients for equilibrium partition of a third element between solid and liquid in iron-carbon base ternary alloys and their relation to graphitization during iron-carbon eutectic solidification. J. Mater. Sci. 19, 2306-2318.

Kurz, W., Fisher, K., 1992. Fundamentals of Solidification, third ed. Trans Tech Publications, pp. 305-306.

Kurz, W., Bezencon, C., Gaumann, M., 2001. Columnar to equiaxed transition in solidification processing. Sci. Technol. Adv. Mater. 2, 185-191.

Lakshminarayanan, A.K., Shanmugam, K., 2009. Effect of autogenous arc welding processes on tensile and impact properties of ferritic stainless steel joints. J. Iron Steel Res. 16, 62-68.

Martorano, M.A., Beckermann, C., Gandin, Ch.A., 2003. A solutal interaction mechanism for the columnar-to-equiaxed transition in alloy solidification. Metall. Mater. Trans. A 34A, 1657-2003.

Yang, Mingbo, Pan, Fusheng, 2009. Analysis about forming mechanism of equiaxed crystal zone for $1 \mathrm{Cr} 18 \mathrm{Ni9Ti}$ stainless steel twin-roll thin strip. J. Mater. Process. Technol. 209, 2203-2211.

Niel, A., Bordreuil, C., Deschaux-Beaume, F., Fras, G., 2013. Modeling hot cracking in 6061 aluminum alloy weld with a microstructural based criterion. Sci. Technol. Weld. Join. 18, 154-160.

Nishi, T., Ohta, H., Shibata, H., Waseda, Y., 2003. Evaluation of the heat leakage in the thermal diffusivity measurement of molten metals by a laser flash method. Int. J. Thermophys. 24, 1735-1751.

Ostrowski, A., Langer, E.W., 1979. Precipitation of titanium carbonitrides in as-cast $17 \%$ chromium stainless steels. Scand. J. Metall. 8, 153-160. 
Petersen, W.A., 1973. Fined grain weld structure. Weld. J. Ind. Technol. 53, 74-79. Rappaz, M., Gandin, C.A., Desbiolles, J.L., Thevoz, P., 1996. Prediction of grain structures in various solidification processes. Metall. Mater. Trans. A 27, 695-705.

Tronche, A., Evans, P.V., Greer, A.L., Bunn, A.M., Bristow, D.J., 2000. Modelling of inoculation of metallic melts: application to grain refinement of aluminium by Al-Ti-B. Acta Mater. 48, 2823-2835.

Villafuerte, J.C., Kerr, H.W., 1990. Grain structures in gas tungsten-arc welds of austenitic stainless steels with ferrite primary phase. Metall. Mater. Trans. A 21, 979-986.
Villafuerte, J.C., Kerr, H.W., David, S.A., 1995. Mechanisms of equiaxed grain formation in ferritic stainless steel gas tungsten arc welds. Mater. Sci. Engineer A 194, 187-191.

Villaret, V., Deschaux-Beaume, F., Bordreuil, C., Fras, G., Chovet, C., Petit, B., 2013a. Characterization of gas metal Arc welding welds obtained with new high $\mathrm{Cr}$-Mo ferritic stainless steel filler wires. Mater. Des. 51, 474-483.

Villaret, V., Deschaux-Beaume, F., Bordreuil, C., Rouquette, S., Chovet, C., 2013b. Influence of filler wire composition on weld microstructures of a 444 ferritic stainless steel grade. J. Mater. Process. Technol. 213, 1538-1547 www.oxford-instruments.com. 